\title{
Basin of Attraction from Modification Variant of Chebyshev-Halley Methods
}

\author{
Hilda Paramita, Sumardi
}

\begin{abstract}
The development of Chebyshev-Halley Method for solving nonlinear equation is presented in this paper. Varian of Chebyshev-Halley method by Xiaojian (2008) was modified using Hermite Interpolation. The convergence analysis shows that these methods have sixth-order convergence for $\beta=0$ and $\beta=1$ eighth-order convergence for $\beta=1 / 2$. The methods are classified by the order and efficiency index. Here, we considered other criteria, the basin of attractions which are presented for several examples.
\end{abstract}

Index Terms: Basin of attraction, Iterative methods, Nonlinear equation, Hermite interpolation.

\section{INTRODUCTION}

Iterative methods are classified by the order of convergence $(p)$ and the number $(d)$ of function (and derivative) evaluation per step. There is efficiency measure defined as,

$$
E=p^{1 / d} \text {. }
$$

Another measure, introduced recently, is the basin of attraction.

Newton's method is the well-known iterative method for finding solution of nonlinear equation $f(x)=0$ by using

$$
x_{n+1}=x_{n}-\frac{f\left(x_{n}\right)}{f^{\prime}\left(x_{n}\right)}, n \in \mathbb{N},
$$

where $f^{\prime}$ is the first derivative of the function $f$. It is known that Newton's method converges quadratically.

Modification of Newton's method is performed to improve the order of convergence so we expect that a new iteration method produces more efficient. The Chebyshev-Halley methods which improve Newton's method are given by

$$
x_{n+1}=x_{n}-\left(1+\frac{1}{2}\left(\frac{L_{f}}{1-\beta x_{n}}\right)\right) \frac{f\left(x_{n}\right)}{f^{\prime}\left(x_{n}\right)},
$$

where

$$
L_{f}=\frac{f\left(x_{n}\right) f^{\prime}\left(x_{n}\right)}{f^{\prime}\left(x_{n}\right)^{2}} .
$$

This family is known to converge cubically and includes, as particular cases, the Chebyshev's method $\beta=0$, Halley's

Revised Manuscript Received on September 22, 2019.

Hilda Paramita, Faculty of Mathematics and Natural Sciences, Universitas Gadjah Mada, Yogyakarta, Indonesia.

Sumardi, Faculty of Mathematics and Natural Sciences, Universitas Gadjah Mada, Yogyakarta, Indonesia. method $\beta=1 / 2$ and super-Halley $\beta=1$.

Xiaojian [1] proposed a variant of Chebyeshev-Halley methods with free from second derivatives and produced equations with fourth-order $(\beta=1 / 2)$ and third-order $(\beta \neq 1 / 2)$

$$
x_{n+1}=x_{n}-\left(\frac{f\left(x_{n}\right)-2 \beta f\left(y_{n}\right)}{f\left(x_{n}\right)-(1+2 \beta) f\left(y_{n}\right)}\right) \frac{f\left(x_{n}\right)}{f^{\prime}\left(x_{n}\right)},
$$

where

$$
y_{n}=x_{n}-\frac{f\left(x_{n}\right)}{f^{\prime}\left(x_{n}\right)} .
$$

Another family of Chebyshev-Halley methods was introduced by Chun [2], Kou, et al [3], Li, et al [4] by using Taylor approximation. Zhao [5] proposed new families of eighth-order methods using some approaches. Hermite Interpolation is an approximation method to modify an iterative method. It was used by Wang and Liu [6] to modify Ostrowski's method with eighth-order.

In recent work, one could find a visual comparison, by plotting the basins of attraction for the methods. Chun et al [7] and Neta et al [8] have developed and compared some iterative methods with high order of convergence by using the basin of attraction.

In the next section, we modified variance of Chebyhev-Halley methods by Xiaojian [1] with Hermite interpolation. Then, a new family of eighth-order methods were presented and analized. We also compared a new method to the existed one by comparing then performance on selected problems using some initial points, the efficiency index and Computational Order of Convergence (COC), and compared it by plotting the basin of attractions for the methods.

\section{THE METHODS AND ANALYSIS OF CONVERGENCE}

We consider the variant of Chebyshev-Halley methods by Xiaojian [1],

$$
\begin{aligned}
& z_{n}=x_{n}-\frac{f\left(x_{n}\right)}{f^{\prime}\left(x_{n}\right)}\left(\frac{f\left(x_{n}\right)-2 \beta f\left(y_{n}\right)}{f\left(x_{n}\right)-(1+2 \beta) f\left(y_{n}\right)}\right), \\
& y_{n}=x_{n}-\frac{f\left(x_{n}\right)}{f^{\prime}\left(x_{n}\right)} .
\end{aligned}
$$




\section{Basin of Attraction from Modification Variant of Chebyshev-Halley Methods}

In obtaining a higher convergence order and a higher efficiency index, we define new step by using Newton's method,

$$
\begin{aligned}
& y_{n}=x_{n}-\frac{f\left(x_{n}\right)}{f^{\prime}\left(x_{n}\right)}, \\
& z_{n}=x_{n}-\frac{f\left(x_{n}\right)}{f^{\prime}\left(x_{n}\right)}\left(\frac{f\left(x_{n}\right)-2 \beta f\left(y_{n}\right)}{f\left(x_{n}\right)-(1+2 \beta) f\left(y_{n}\right)}\right), \\
& x_{n+1}=z_{n}-\frac{f\left(z_{n}\right)}{f^{\prime}\left(z_{n}\right)} .
\end{aligned}
$$

To derive a scheme with a higher convergence order, we approximate $f^{\prime}\left(z_{n}\right)$ using a Hermite interpolation. We construct a Hermite interpolation polynomial $H_{3}(x)$, that needs to meet the interpolation conditions

$$
\begin{aligned}
& H_{3}\left(x_{n}\right)=f\left(x_{n}\right) ; H_{3}\left(y_{n}\right)=f\left(y_{n}\right) ; \\
& H_{3}\left(z_{n}\right)=f\left(z_{n}\right) ; H_{3}^{\prime}\left(x_{n}\right)=f^{\prime}\left(x_{n}\right) .
\end{aligned}
$$

Then $H_{3}(x)$ can be written as follows,

$$
\begin{aligned}
H_{3}(x) & =a_{0}(x) f\left(x_{n}\right)+a_{1}(x) f\left(y_{n}\right)+a_{2}(x) f\left(z_{n}\right) \\
+ & a_{0}(x) f^{\prime}\left(x_{n}\right),
\end{aligned}
$$

where $a_{0}(x), a_{1}(x), a_{2}(x), \overline{a_{0}(x)}$ are cubic polynomial functions satisfying the following conditions

(i) $a_{0}\left(x_{n}\right)=1 ; a_{1}\left(x_{n}\right)=0 ; a_{2}\left(x_{n}\right)=0 ; \overline{a_{0}\left(x_{n}\right)}=0$,

(ii) $a_{0}\left(x_{n}\right)=0 ; a_{1}\left(x_{n}\right)=1 ; a_{2}\left(x_{n}\right)=0 ; \overline{a_{0}\left(x_{n}\right)}=0$,

(iii) $a_{0}\left(x_{n}\right)=0 ; a_{1}\left(x_{n}\right)=0 ; a_{2}\left(x_{n}\right)=1 ; \overline{a_{0}\left(x_{n}\right)}=0$,

(iv) $a_{0}\left(x_{n}\right)=0 ; a_{1}\left(x_{n}\right)=0 ; a_{2}\left(x_{n}\right)=0 ; \overline{a_{0}\left(x_{n}\right)}=0$.

From above conditions, we have

$$
\begin{aligned}
& H_{3}(x)=\frac{\left(x-y_{n}\right)\left(x_{n}-z_{n}\right)}{\left(x_{n}-y_{n}\right)\left(x_{n}-z_{n}\right)}\left[1-\frac{\left(x-x_{n}\right)\left(2 x_{n}-y_{n}-z_{n}\right)}{\left(x_{n}-y_{n}\right)\left(x_{n}-z_{n}\right)^{2}}\right] f\left(x_{n}\right) \\
& +\frac{\left(x-z_{n}\right)\left(x-x_{n}\right)^{2}}{\left(y_{n}-x_{n}\right)^{2}\left(y_{n}-z_{n}\right)} f\left(y_{n}\right)+\frac{\left(x-x_{n}\right)^{2}\left(x-y_{n}\right)}{\left(z_{n}-x_{n}\right)^{2}\left(z_{n}-y_{n}\right)} f\left(z_{n}\right) \\
& +\frac{\left(x-x_{n}\right)\left(x-y_{n}\right)\left(x-z_{n}\right)}{\left(x_{n}-y_{n}\right)\left(x_{n}-z_{n}\right)} f^{\prime}\left(x_{n}\right),
\end{aligned}
$$

Then by simplifying, we have

$$
\begin{aligned}
& H_{3}^{\prime}\left(z_{n}\right)=2 f\left[x_{n}, z_{n}\right]+f\left[y_{n}, z_{n}\right]-2 f\left[x_{n}, y_{n}\right] \\
& \quad+\left(y_{n}-z_{n}\right) f\left[y_{n}, x_{n}, x_{n}\right],
\end{aligned}
$$

where

$$
\begin{aligned}
& f\left[x_{n}, z_{n}\right]=\frac{f\left(z_{n}\right)-f\left(x_{n}\right)}{z_{n}-x_{n}} ; \\
& f\left[x_{n}, y_{n}\right]=f\left[y_{n}, x_{n}\right]=\frac{f\left(y_{n}\right)-f\left(x_{n}\right)}{y_{n}-x_{n}} ; \\
& f\left[y_{n}, z_{n}\right]=\frac{f\left(z_{n}\right)-f\left(y_{n}\right)}{z_{n}-y_{n}} \\
& f\left[y_{n}, x_{n}, x_{n}\right]=\frac{f\left[y_{n}, x_{n}\right]-f^{\prime}\left(x_{n}\right)}{y_{n}-x_{n}}
\end{aligned}
$$

We obtain an approximation of $f^{\prime}\left(z_{n}\right)$,

$$
\begin{aligned}
f^{\prime}\left(z_{n}\right) \approx H_{3}^{\prime}\left(z_{n}\right) & =2 f\left[x_{n}, z_{n}\right]+f\left[y_{n}, z_{n}\right]-2 f\left[x_{n}, y_{n}\right] \\
& +\left(y_{n}-z_{n}\right) f\left[y_{n}, x_{n}, x_{n}\right] .
\end{aligned}
$$

Therefore, we obtain a new family of Chebyshev-Halley method as follows,

$$
\begin{aligned}
y_{n} & =x_{n}-\frac{f\left(x_{n}\right)}{f^{\prime}\left(x_{n}\right)}, \\
z_{n} & =x_{n}-\frac{f\left(x_{n}\right)}{f^{\prime}\left(x_{n}\right)}\left(\frac{f\left(x_{n}\right)-2 \beta f\left(y_{n}\right)}{f\left(x_{n}\right)-(1+2 \beta) f\left(y_{n}\right)}\right), \\
x_{n+1} & =z_{n}-\frac{f\left(z_{n}\right)}{2 f\left[x_{n}, z_{n}\right]+f\left[y_{n}, z_{n}\right]-2 f\left[x_{n}, y_{n}\right]+\left(y_{n}-z_{n}\right) f\left[y_{n}, x_{n}, x_{n}\right]},
\end{aligned}
$$

where $\beta \in \mathbb{R}$. The best choice of $\beta$ is shown in the following theorem.

Theorem 1. Let $\alpha \in I$ be a simple zero of sufficiently differentiable function $f: I \rightarrow R$ for an open interval $I$. If $x_{0}$ is sufficiently close to $\alpha$, then the methods defined by (9) have sixth-order for any $\beta \in \mathbb{R}$ and eighth-order for $\beta=1 / 2$, and satisfy the error equation

$$
\begin{aligned}
& e_{n+1}=(2 \beta-1)^{2} c_{2}^{5} e_{n}^{6}-(2 \beta-1)\left(8 c_{2}^{3} \beta^{2}-20 \beta c_{2}^{3}\right. \\
& \left.\quad+16 \beta c_{2} c_{3}-5 c_{2} c_{3}-c_{4}+4 c_{2}^{3}\right) c_{2}^{3} e_{n}^{7}+\left(4 c_{2}^{2} \beta^{2}-10 \beta c_{2}^{2}\right. \\
& \left.\quad+8 \beta c_{3}+3 c_{2}^{2}-3 c_{3}\right)\left(4 c_{2}^{3} \beta^{2}-10 \beta c_{2}^{3}+8 \beta c_{2} c_{3}+c_{2}^{3}\right. \\
& \left.\quad-2 c_{2} c_{3}-c_{4}\right) c_{2}^{2} e_{n}^{8}+O\left(e_{n}^{9}\right) .
\end{aligned}
$$

Proof. Let $c_{j}=\frac{1}{j !} \frac{f^{(j)}(\alpha)}{f^{\prime}(\alpha)}, j=1,2,3, \ldots$ and $e_{n}=x_{n}-\alpha$. By using Taylor expansion around $x_{n}=\alpha$ and taking into account $f(\alpha)=0$, we have

$$
f\left(x_{n}\right)=f^{\prime}(\alpha)\left(e_{n}+c_{2} e_{n}^{2}+c_{3} e_{n}^{3}+c_{4} e_{n}^{4}+O\left(e_{n}^{5}\right)\right),
$$
and

$$
f^{\prime}\left(x_{n}\right)=f^{\prime}(\alpha)\left(1+2 c_{2} e_{n}+3 c_{3} e_{n}^{2}+4 c_{4} e_{n}^{3}+O\left(e_{n}^{4}\right)\right) .
$$

Dividing (11) and (12), we have

$$
\begin{aligned}
& \frac{f\left(x_{n}\right)}{f^{\prime}\left(x_{n}\right)}=e_{n}-c_{2} e_{n}^{2}+2\left(c_{2}^{2}-c_{3}\right) e_{n}^{3}+\left(7 c_{2} c_{3}-4 c_{2}^{3}\right. \\
& \left.\quad-3 c_{4}\right) e_{n}^{4}+O\left(e_{n}^{5}\right), \\
& f\left(y_{n}\right)=f^{\prime}(\alpha)\left(c_{2} e_{n}^{2}+2\left(c_{2}^{2}+c_{3}\right) e_{n}^{3}+\left(3 c_{4}-3 c_{2}^{3}\right.\right. \\
& \left.\left.\quad-7 c_{2} c_{3}\right) e_{n}^{4}+\cdots+O\left(e_{n}^{9}\right)\right),
\end{aligned}
$$

so

$$
\begin{aligned}
z_{n} & =\alpha+(2 \beta-1) c_{2}^{2} e_{n}^{3}-\left(4 \beta^{2} c_{2}^{3}-10 \beta c_{2}^{3}+3 c_{2}^{3}\right. \\
& \left.+8 \beta c_{2} c_{3}-3 c_{2} c_{3}\right) e_{n}^{4}+O\left(e_{n}^{5}\right) .
\end{aligned}
$$

Again, by using Taylor expansion of $f\left(z_{n}\right)$ about $\alpha$ it gives,

$$
\begin{gathered}
f\left(z_{n}\right)=f^{\prime}(\alpha)\left(c_{2}^{2}(2 \beta-1) e_{n}^{3}+\left(3 c_{2} c_{3}-8 \beta c_{2} c_{3}-3 c_{2}^{3}\right.\right. \\
\left.\left.-4 \beta^{2} c_{2}^{3}+10 \beta c_{2}^{3}\right) e_{n}^{4}+\cdots+O\left(e_{n}^{9}\right)\right)
\end{gathered}
$$

Then, we have 


$$
\begin{aligned}
& f\left[x_{n}, z_{n}\right]=f^{\prime}(\alpha)\left(1+c_{2} e_{n}+c_{3} e_{n}^{2}+\left(c_{4}-c_{2}^{3}+2 \beta c_{2}^{3}\right) e_{n}^{3}\right. \\
& \quad+\left(10 \beta c_{2}^{4}-3 c_{2}^{4}-6 \beta c_{2}^{2} c_{3}+2 c_{3} c_{2}^{2}-4 \beta^{2} c_{2}^{4}\right) e_{n}^{4} \\
& \left.\quad+O\left(e_{n}^{5}\right)\right), \\
& f\left[y_{n}, z_{n}\right]=f^{\prime}(\alpha)\left(1+c_{2}^{2} e_{n}+\left(2 c_{2} c_{3}+2 \beta c_{2}^{3}-3 c_{2}^{3}\right) e_{n}^{3}\right. \\
& \quad+\left(-4 \beta^{2} c_{2}^{4}+10 \beta c_{2}^{4}-4 c_{3} c_{2}^{2}+3 c_{2} c_{3}^{4}-7 c_{2}^{4}\right. \\
& \left.\left.\quad-8 \beta c_{2}^{2} c_{3}\right) e_{n}^{4}+O\left(e_{n}^{5}\right)\right), \\
& f\left[x_{n}, y_{n}\right]=f^{\prime}(\alpha)\left(1+c_{2} e_{n}+\left(c_{2}^{2}+c_{3}\right) e_{n}^{2}+\left(c_{4}-2 c_{2}^{3}\right.\right. \\
& \left.\quad+3 c_{2} c_{3}\right) e_{n}^{3}+\left(4 c_{2} c_{3}-8 c_{3} c_{2}^{2}+2 c_{3}^{2}-5 c_{2}^{4}\right) e_{n}^{4} \\
& \left.\quad+O\left(e_{n}^{5}\right)\right), \\
& \left(y_{n}-z_{n}\right) f\left[y_{n}, x_{n}, x_{n}\right]=f f^{\prime}(\alpha)\left(c_{2}^{2}+\left(4 c_{2} c_{3}-c_{2}^{3}\right.\right. \\
& \left.\quad-2 \beta c_{2}^{3}\right) e_{n}^{3}+\left(4 c_{3}^{2}+4 \beta c_{2}^{2} c_{3}+6 c_{2} c_{4}+4 \beta^{2} c_{2}^{4}\right. \\
& \left.\left.\quad-11 c_{3} c_{2}^{2}-c_{2}^{4}-10 \beta c_{2}^{4}\right) e_{n}^{4}+O\left(e_{n}^{5}\right)\right) .
\end{aligned}
$$

Using equation (16) and (17), we have

$$
\begin{aligned}
& \frac{f\left(z_{n}\right)}{2 f\left[x_{n}, z_{n}\right]+f\left[y_{n}, z_{n}\right]-2 f\left[x_{n}, y_{n}\right]+\left(y_{n}-z_{n}\right) f\left[y_{n}, x_{n}, x_{n}\right]} \\
& =(2 \beta-1) c_{2}^{2} e_{n}^{3}+\left(4 \beta-4 \beta^{2}-1\right) c_{2}^{5} e_{n}^{6}+\cdots+O\left(e_{n}^{9}\right) .
\end{aligned}
$$

By substituting (15) and (18) in (9) and simplifying, we have

$$
\begin{aligned}
& e_{n+1}=(2 \beta-1)^{2} c_{2}^{5} e_{n}^{6}-(2 \beta-1)\left(8 \beta^{2} c_{2}^{3}-20 \beta c_{2}^{3}\right. \\
& \left.\quad+16 \beta c_{2} c_{3}-5 c_{2} c_{3}-c_{4}+4 c_{2}^{3}\right) c_{2}^{3} e_{n}^{7}+\left(4 \beta^{2} c_{2}^{2}\right. \\
& \left.-10 \beta c_{2}^{2}+8 \beta c_{3}+3 c_{2}^{2}-3 c_{3}\right)\left(4 \beta^{2} c_{2}^{3}-10 \beta c_{2}^{3}\right. \\
& \left.+8 \beta c_{2} c_{3}+c_{2}^{3}-2 c_{2} c_{3}-c_{4}\right) c_{2}^{2} e_{n}^{8}+O\left(e_{n}^{9}\right) .
\end{aligned}
$$

It means that the method defined by (9) is at least of order six for any $\beta$.

It's obvious that $2 \beta-1=0$, that is $\beta=1 / 2$, then the error equation should be

$$
e_{n+1}=\left(-5 c_{2}^{5} c_{3}+3 c_{2}^{7}+2 c_{2}^{3} c_{3}^{2}+c_{4} c_{2}^{4}-c_{3} c_{2}^{2} c_{4}\right) e_{n}^{8}+O\left(e_{n}^{9}\right) .
$$

Which means that the method defined by (9) is eighth-order if $\beta=1 / 2$.

Per iteration, the present methods require three evaluations of the functions, one of them is its first derivative. We consider the definition of efficiency index as $p^{1 / d}$, where $p$ is the order of the method and $d$ is the number of functions evaluations per iteration required by the method. The new method has the efficiency index of $6^{1 / 4} \approx 1.565$ with any $\beta$ and $8^{1 / 4} \approx 1.682$ with $\beta=1 / 2$, which is better than $3^{1 / 3} \approx 1.442$ in [1] and Newton's method $2^{1 / 2} \approx 1.414$ in [6].

\section{NUMERICAL EXAMPLES}

In this section, we present numerical results on some tests function to compare the efficiency of the proposed family of methods with some known methods.

\section{A. Computational Order of Convergence}

Numerical computation reported here have been carried out in the Maple software with 800 significant digits. We use the stopping criteria for the iterative process error $=\left|x_{n}-x_{n-1}\right|<\varepsilon$, where $\varepsilon=10^{-40}$. The computational order of convergence (COC) denoted as $\rho$ is given by (see

\begin{tabular}{|c|c|c|c|c|c|c|c|c|c|c|c|c|c|c|c|}
\hline \multirow{2}{*}{$\begin{array}{l}\text { Iterative } \\
\text { Methods }\end{array}$} & \multirow[t]{2}{*}{$N$} & $e$ & $\rho$ & $N$ & $e$ & $\rho$ & $N$ & $e$ & $\rho$ & $N$ & $e$ & $\rho$ & $N$ & $e$ & $\rho$ \\
\hline & & \multicolumn{2}{|c|}{$f_{1}(x), x_{0}=3.1$} & \multicolumn{3}{|c|}{$f_{2}(x), x_{0}=-1.3$} & \multicolumn{3}{|c|}{$f_{3}(x), x_{0}=2.0$} & \multicolumn{3}{|c|}{$f_{4}(x), x_{0}=1.0$} & \multicolumn{3}{|c|}{$f_{5}(x), x_{0}=1.9$} \\
\hline VMCH1 & 7 & $1.9 \mathrm{e}-631$ & 2.99 & 6 & $3.2 \mathrm{e}-$ & 2.99 & 6 & 9.1e- & 3.00 & 6 & $4.1 \mathrm{e}-271$ & 3.00 & 6 & $1.1 \mathrm{e}-404$ & 3.00 \\
\hline $\mathrm{VMCH} 2$ & 5 & $5.9 \mathrm{e}-408$ & 3.99 & 4 & $1.1 \mathrm{e}-294$ & 4.00 & 5 & $6.8 \mathrm{e}-469$ & 3.99 & 5 & $2.4 \mathrm{e}-525$ & 3.99 & 5 & 694 & 3.99 \\
\hline VMCH3 & 8 & $6.1 \mathrm{e}-537$ & 3.00 & 6 & $1.8 \mathrm{e}-603$ & 3.00 & 6 & $3.8 \mathrm{e}-318$ & 3.00 & 6 & $4.2 \mathrm{e}-305$ & 3.00 & 6 & $1.4 \mathrm{e}-428$ & 3.00 \\
\hline MVMCH1 & 4 & $9.1 \mathrm{e}-362$ & 5.99 & 3 & $1.5 \mathrm{e}-186$ & 5.99 & 4 & $1.2 \mathrm{e}-524$ & 5.99 & 4 & $5.3 e-487$ & 5.99 & 4 & $3.4 \mathrm{e}-722$ & 5.99 \\
\hline MVMCH2 & 3 & $8.4 \mathrm{e}-164$ & 7.99 & 3 & $2.3 e-506$ & 8.00 & 3 & $1.2 \mathrm{e}-256$ & 7.99 & 3 & $1.3 \mathrm{e}-244$ & 7.99 & 3 & $2.7 \mathrm{e}-331$ & 7.99 \\
\hline MVMCH3 & 4 & $6.1 \mathrm{e}-151$ & 6.00 & 3 & $3.1 \mathrm{e}-181$ & 6.00 & 4 & $1.2 \mathrm{e}-578$ & 5.99 & 4 & $6.4 \mathrm{e}-540$ & 6.00 & 4 & $1.0 \mathrm{e}-758$ & 6.00 \\
\hline
\end{tabular}
[9]) :

$$
\rho_{n} \approx \frac{\ln \left|\left(x_{n+1}-\alpha\right) /\left(x_{n}-\alpha\right)\right|}{\ln \left|\left(x_{n}-\alpha\right) /\left(x_{n-1}-\alpha\right)\right|} .
$$

Based on Wang and Liu [6], we use the following functions and their simple zeroes given as the following:

$$
\begin{array}{lll}
f_{1}(x)=e^{x^{2}+7 x-30}-1, & \alpha=3 \\
f_{2}(x)=x e^{x^{2}}-\sin ^{2} x+3 \cos x+5, & \alpha=-1.207644782 \cdots \\
f_{3}(x)=10 x e^{-x^{2}}-1, & \alpha=1.6796306104 \cdots \\
f_{4}(x)=x^{5}+x^{4}+4 x^{2}-15, & \alpha=1.3473280998 \cdots \\
f_{5}(x)=(x-1)^{6}-1, & \alpha=2 .
\end{array}
$$

We compare variance of Chebyshev-Halley methods by Xiaojian [8] defined as variant of Chebyshev-Halley with $\beta=1$ (VMCH1) and its modification (MVMCH1), variant of Chebyshev-Halley with $\beta=1 / 2(\mathrm{VMCH} 2)$ and its modification (MVMCH2) and variant of Chebyshev-Halley with $\beta=0(\mathrm{VMCH} 3)$ and its modification (MVMCH3).

Generally, from Table I, the modification has increased the order of convergence. Then, it can be seen that MVMCH2 has the highest order of convergence based on computational order of convergence (COC) and converge in the lowest number of iterations with smallest error when compared to the other methods.

Table I . Value of COC from Variant of Chebyshev Halley Method and its Modification 


\section{Basin of Attraction from Modification Variant of Chebyshev-Halley Methods}

\section{A. Basin of Attractions}

In previous discussion, we analyzed the methods whose roots are in the real domain $f: D \subset \mathbb{R} \rightarrow \mathbb{R}$. It can be extended to functions defined in the complex plane $f: D \subset \mathbb{C} \rightarrow \mathbb{C}$ by using basin of attraction. The basin of attraction is a method used to usually understand how a method behaves as a function of the various starting points is defined as the basin of attraction. All the examples have roots within a square of $[-3,3] \times[-3,3]$. In this region, we have 360.000 equally spaced points with mesh $h=0.01$. Each grid point is used as initial points for the methods and we have registered the total number of iterations required to converge to a root and also to which root it converges. Every point is a form of a complex number, that is $z=x_{k}+i y_{j}$ where $j, k \in \mathbb{N}$.

1. $f(\mathrm{z})=\mathrm{z}^{2}-1$

The polynomial has two different real roots, $\alpha_{1}=-1$ and $\alpha_{2}=1$. We used a different color for each basin, so that we could tell if the method converge to the closest root. In this case, the red color is the set all of initial values converge to $\alpha_{1}$ and the blue color is the set all of initial values converge to $\alpha_{2}$ in at most 40 iterations or the stopping criteria for the iteration $\left|z_{n}-\alpha_{j}\right|<10^{-5}, n \in \mathbb{N}$ where $j=1,2$. The black regions correspond to initial points for which the iteration fails to converge. Fig. 1 shows comparison of basin of attraction between variance of Chebyeshev-Halley method and its modification that these methods have a good performance because all starting points are converging.

(a)

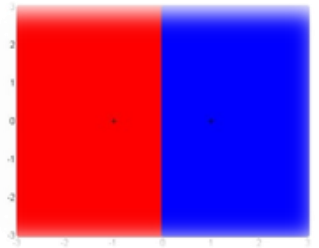

(c)

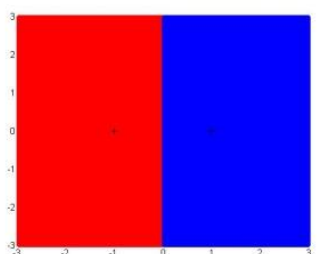

(e)

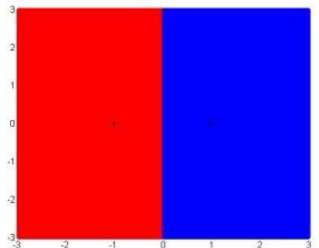

(b)

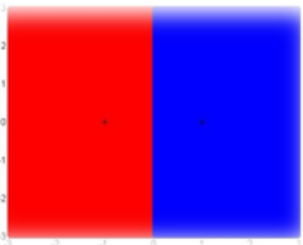

(d)

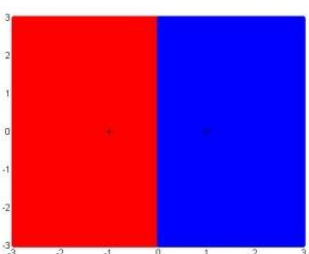

(f)

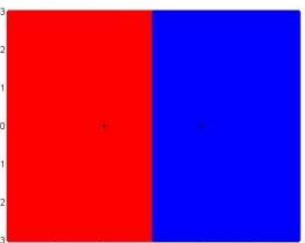

Fig. 1. Basin of attraction for (a) VMCH1, (b) $\mathrm{VMCH} 2$, (c) VMCH3, (d) MVMCH1, (e) MVMCH2 and (f) MVMCH3 applied to $f(z)=z^{2}-1$

\section{2. $f(\mathrm{z})=\mathrm{z}^{3}-1$}

The polynomial has a real root and two complex roots, they are $\quad \alpha_{1}=1, \quad \alpha_{2}=-0.5+0.866025403784439 i \quad$ and $\alpha_{3}=-0.5-0.866025403784439 i$. We used a different color for each basin. We assigned green color if they converge to $\alpha_{1}$, red color if they converge to $\alpha_{2}$ and blue color if they converge to $\alpha_{3}$ in at most 40 iterations and if $\left|z_{n}-\alpha_{j}\right|<10^{-5}, n \in \mathbb{N}$ where $j=1,2,3$.

Fig. 2 shows comparison of basin of attractions between variance of Chebyshev-Halley method and its modification. The black regions correspond to initial points for which the iteration fails to converge. Viewing from picture (a) and (d) for $\beta=0$, it can be said that performance of variance of Chebyshev-Halley method has an improvement after being modified. There are diverging points for the method of variance of Chebyshev-Halley methods for $\beta=0$. Generally, based on basin of attraction for $f(z)=z^{3}-1$, variance of Chebyshev-Halley methods shows an improvement after being modified using Hermite interpolation. (a)

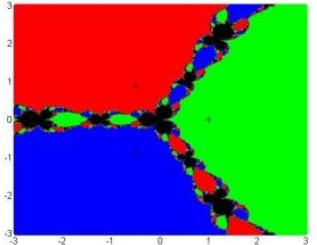

(c)

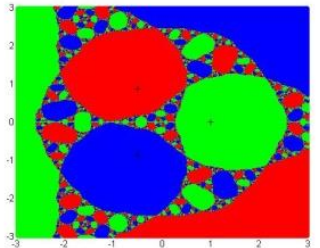

(e)

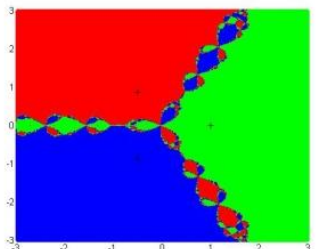

(b)

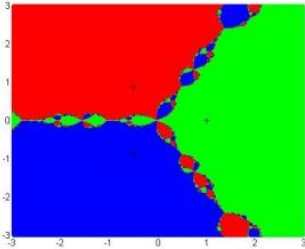

(d)

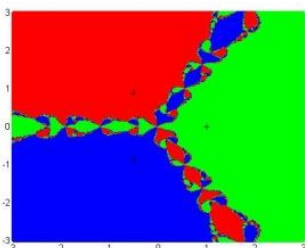

(f)

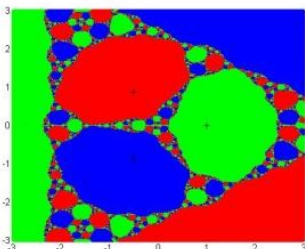

Fig. 2. Basin of attraction for (a) VMCH1, (b) $\mathrm{VMCH} 2$, (c) VMCH3, (d) MVMCH1, (e) MVMCH2 and

(f) MVMCH3 applied to $f(z)=z^{3}-1$

\section{3. $f(\mathrm{z})=\mathrm{z}^{4}-1$}

This polynomial has two real roots and two imaginary roots, they are $\alpha_{1}=-1, \alpha_{2}=i, \alpha_{3}=-i$ and $\alpha_{4}=1$. In this case, we assigned red color if they converge to $\alpha_{1}$, blue color if they converge to $\alpha_{2}$, green color if they converge to $\alpha_{3}$ and cyan color if they converge to $\alpha_{4}$ in at most 40 iterations and if $\left|z_{n}-\alpha_{j}\right|<10^{-5}, n \in \mathbb{N}$ where $j=1,2,3,4$.

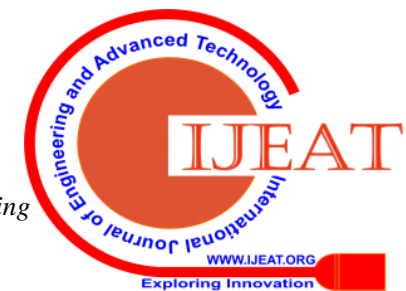


Fig. 3 shows comparison of basin of attractions between variant of Chebyshev-Halley method and its modification. The black regions correspond to initial points for which the iteration fails to converge. Clearly, by looking at fig. (a), (d) and (c), (f), then variant of Chebyshev-Halley method has an improvement after being modified. Because, fig. (d) has no black region as much as fig. (a). Roughly fig. (c) has more black regions, it means that based on basin of attractions for polynomial $f(z)=z^{4}-1$, modification of variance of Chebyshev-Halley method has less black region which initial points make the iteration fails to converge. (a)

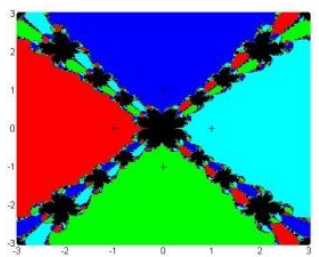

(c)

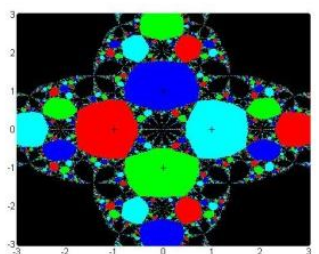

(e)

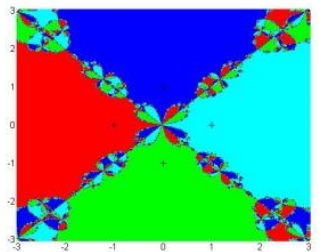

(b)

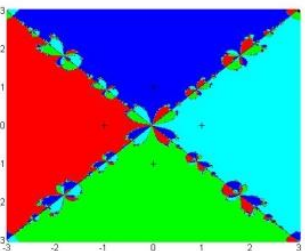

(d)

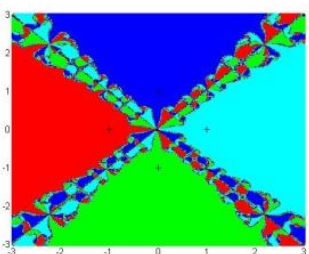

(f)

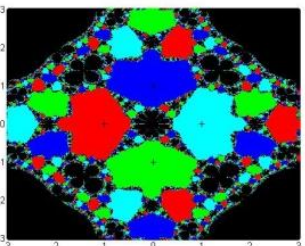

Fig. 3. Basin of attraction for (a) $\mathrm{VMCH} 1$, (b) $\mathrm{VMCH} 2$, (c) VMCH3, (d) MVMCH1, (e) MVMCH2 and (f) $\mathrm{MVMCH} 3$ applied to $f(z)=z^{4}-1$

\section{4. $f(\mathrm{z})=\mathrm{z}^{4}-10 \mathrm{z}^{2}+9$}

The polynomial has four different roots, they are $\alpha_{1}=-3$, $\alpha_{2}=3, \alpha_{3}=-1$ and $\alpha_{4}=1$. In this case we assign red color if they converge to $\alpha_{1}$, blue color if they converge to $\alpha_{2}$, green color if they converge to $\alpha_{3}$ and cyan color if they converge to $\alpha_{4}$ in at most 40 iterations and if $\left|z_{n}-\alpha_{j}\right|<10^{-5}, n \in \mathbb{N}$ where $j=1,2,3,4$. Fig. 4 shows comparison of basin of attraction between variance of Chebyshev-Halley methods and its modification with $f(z)=z^{4}-10 z^{2}+9$. The black regions correspond to initial points for which the iteration fails to converge.

The differences between fig. (a), (d) and (b), (e) showed less significant difference, but at (a), it is apparently seen that there are (very small) black regions than fig. (d). Furthermore, looking at fig. (c) and (f), we can see that variance of Chebyshev-Halley method has more black regions that its modification, which cause variance of
Chebyshev-Halley method has an improvement after being modified using Hermite interpolation. (a)

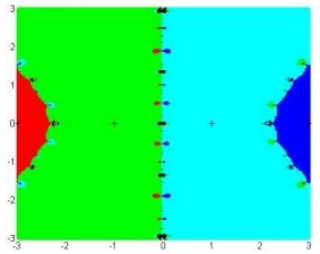

(c)

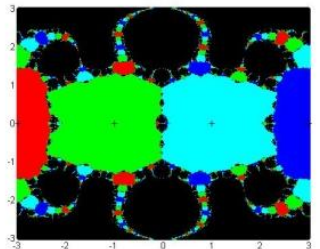

(e)

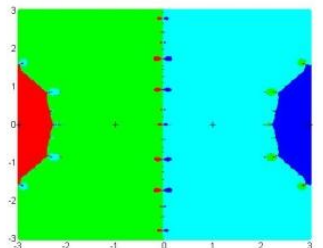

(b)

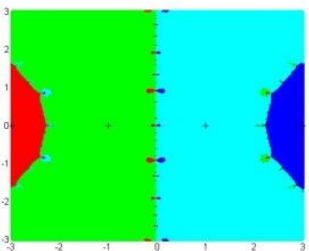

(d)

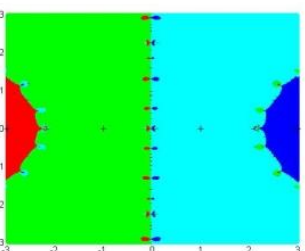

(f)

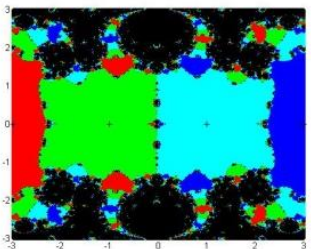

Fig. 4. (a) VMCH1, (b) VMCH2, (c) VMCH3, (d) MVMCH1, (e) MVMCH2 and (f) MVMCH3 applied to

$$
f(z)=z^{4}-10 z^{2}+9
$$

5. $f(\mathbf{z})=\mathbf{z}^{3}-\mathbf{z}$

The polynomial has three real roots, they are $\alpha_{1}=0$, $\alpha_{2}=-1$ and $\alpha_{3}=1$. In this case, we assigned red color if they converge to $\alpha_{1}$, blue color if they converge to $\alpha_{2}$ and green color if the converge to $\alpha_{3}$ in at most 40 iterations and if $\left|z_{n}-\alpha_{j}\right|<10^{-5}, n \in \mathbb{N} \quad$ where $j=1,2,3$. The black regions correspond to initial points for which the iteration fails to converge. Looking at Fig. 5, it shows comparison between variance of Chebyshev-Halley methods and its modification. The difference in fig. (a), (d) and (b), (e) do not show a significant difference. Furthermore, looking at fig. (c) and (f), it can be seen that variance of Chebyshev-Halley method has more black regions than its modification. It shows that its modification is better after being modified using Hermite interpolation.

Using Matlab, we have also collected the time (in seconds) required to run each method on all initial points. Table II shows that variance of Chebyshev-Halley method (VMCH2) and its modification (MVMCH2) with $\beta=1 / 2$ take a relatively short time than other methods. Afterwards, variance of modification of the Chebyshev-Halley with $\beta=1$ takes longer time than the others. It can be seen that if we review from different initial values, the higher of order of convergence can not always guarantee the performance of the method which is better than others with the lower of order of convergence. 
(a)

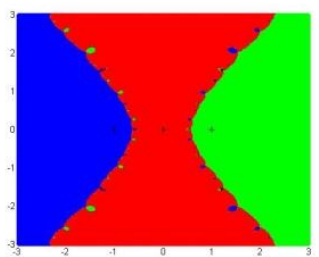

(c)

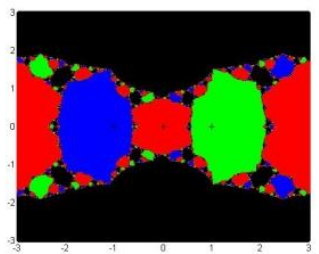

(e)

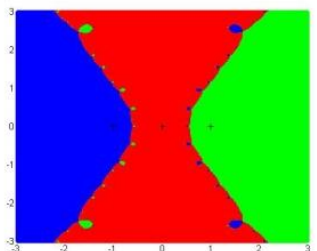

(b)

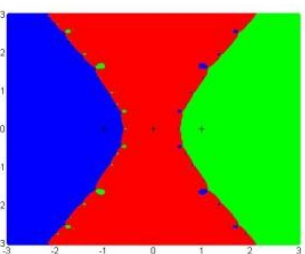

(d)

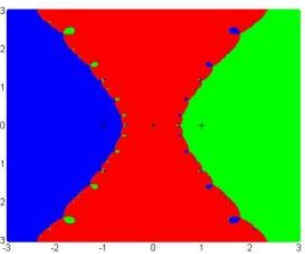

(f)

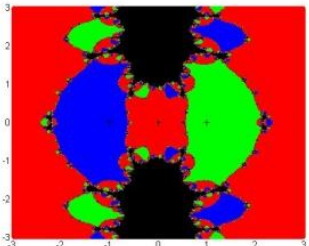

(d) MVMCH1, (e) MVMCH2 and (f) MVMCH3 applied to

$$
f(z)=z^{3}-z
$$

\section{CONCLUSION}

As a whole, the above comparison showed that variance modification of the Chebyshev-Halley method was better than others. It can be seen from pattern made by index of efficiency, COC and the basin of attraction of the method. Afterwards, the modification of the method with $\beta=1 / 2$ was considered as the best one seen by the needed amount of the iteration and order of convergence which are higher than other method. So it can be concluded that variance of the Chebyshev-Halley method and its modification with $\beta=1 / 2$ are the best iteration method.

\section{ACKNOWLEDGMENT}

This research was supported by Research Grant PDUPT contract number 2557/UN1.DITLIT/DIT-LIT/LT/2019 provided by the Directorate General of the Higher Education of the Republic of Indonesia. The authors thank the anonymous referees for their valuable suggestions and comments.

Fig. 5. (a) $\mathrm{VMCH} 1$, (b) $\mathrm{VMCH} 2$, (c) $\mathrm{VMCH} 3$,

Table II: Computational time for basin of attraction

\begin{tabular}{lccccccc}
\hline $\begin{array}{c}\text { Iterative } \\
\text { methods }\end{array}$ & $\begin{array}{c}\text { Order of } \\
\text { convergence }\end{array}$ & $f_{1}$ & $f_{2}$ & $f_{3}$ & $f_{4}$ & $f_{5}$ & $\begin{array}{c}\text { Average } \\
\text { times }(\boldsymbol{s})\end{array}$ \\
\hline VMCH1 & 3 & 350.879 & 385.185 & 437.647 & 377.238 & 461.329 & 402.0556 \\
VMCH2 & 4 & 340.116 & 363.039 & 378.358 & 355.226 & 445.435 & 376.4348 \\
VMCH3 & 3 & 334.737 & 364.887 & 539.918 & 472.211 & 763.563 & 495.0623 \\
MVMCH1 & 6 & 361.285 & 373.130 & 447.251 & 374.673 & 506.882 & 412.6442 \\
MVMCH2 & 8 & 342.994 & 362.313 & 412.270 & 367.521 & 491.849 & 397.1894 \\
MVMCH3 & 6 & 346.882 & 388.467 & 836.737 & 532.952 & 722.689 & 565.5454 \\
\hline
\end{tabular}

\section{REFERENCES}

1. Xiaojian, Zhou, "Modified Chebyshev-Halley Methods Free from Second Derivative", Applied Mathematics and Computation, vol. 203, pp. 824-827, 2008.

2. Chun, Changbum, "Some Variants of Chebyshev-Halley Methods Free from Second Derivative", Applied Mathematics and Computation, vol. 191, pp.193-198, 2007.

3. Kou, Jisheng., and Li, Yitian, "Modified Chebyshev-Halley Methods with Sixth-order Convergence", Applied Mathematics and Computation, vol. 188, pp.681-685, 2007.

4. Li, Yaotang., et al, "Some New Variants of Chebyshev-Halley Methods Free from Second Derivative", International Journal of Nonlinear Science, vol. 9, (2), pp. 201-206, 2010.

5. Zhao, Lingling., et al, "New Families of Eighth-Order Methods with High Efficiency Index for Solving Nonlinear Equations", WSEAS Transactions on Mathematics, vol. 11, pp. 283-293, 2012.

6. Wang, Xia., and L. Liu, "Modified Ostrowski's Method with Eighth-Order Convergence and High Efficiency Index", Applied Mathematics Letters, vol. 23, pp. 549-554, 2010.

7. Chun, Changbum, "Some Variants of King's Fourth-Order Family of Methods for Nonlinear Equations", Applied Mathematics and Computation, vol. 190, pp. 57-62, 2007

8. Neta, Beny., Scott, Melvin., and Chun, Changbum, "Basin of Attractions for Several Methods to Find Simple Roots of Nonlinear Equations", Applied Mathematics and Computations, vol. 218, pp.10548-10556, 2012.

9. L.O. Jay, “A note on q-order of convergence”, BIT 41, pp 422-429, 2001.

\section{Authors Profile}

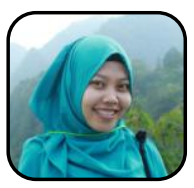

Hilda Paramita is a mathematics teacher who was a graduate student in mathematics, Faculty of Science and Technology, State Islamic University of Sultan Syarif Kasim, Pekanbaru. At that time, she had the opportunity to become an assistant lecturer at the level of two courses. Then, she continued his education and became a graduate student in mathematics, Faculty of Mathematics and Natural Sciences, Universitas Gadjah Mada, Yogyakarta. During the college, to complete her education as a student, she was interested in conducting research in the numerical method field i.e. modification of the iteration equation to solve nonlinear equations.

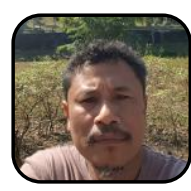

Dr. Sumardi, M.Si is Associate Professor at Gadjah Mata University, Yogyakarta, Indonesia. He has 21 years of experience in teaching in undergraduate, 7 years of experience in teaching in Graduate level and 19 years of experience in Research. He has received M.Si from Institute of Bandung Technology, Bandung, Indonesia, 1998 and Dr. from Gadjah Mada University, Yogyakarta, Indonesia, 2012. Presently he is Head of the Computational Mathematics Laboratory at Department of Mathematics, Gadjah Mada University. His Research area is Computational Mathematics, Numerical Analysis, Ordinary and Partial Differential Equation. 